\title{
EL TRATADO DE NO PROLIFERACIÓN DE ARMAS NUCLEARES: LOS TEMAS CLAVE EN LA CONFERENCIA DE EXAMEN EN 2015
}

\author{
Gonzalo de Salazar ${ }^{1}$ \\ Embajador de España
}

\begin{abstract}
Resumen:
El régimen de no proliferación nuclear basado durante cuarenta y cinco años en el Tratado de No Proliferación de armas nucleares (TNP) se enfrenta actualmente a importantes desafíos. En las últimas décadas varios estados han desarrollado armas nucleares, permaneciendo al margen del Tratado, mientras otros han desarrollado capacidades nucleares significativas, dando lugar a tensiones regionales. Por otra parte, se ha producido un renacimiento de la energía nuclear como respuesta a nuevas necesidades energéticas, dando lugar a un creciente desarrollo de la tecnología nuclear en el mundo. Dado el carácter dual civil y militar de la tecnología nuclear, este proceso es un reto importante para la comunidad internacional, que debe perseverar en el mantenimiento de un equilibrio entre el progreso técnico y económico y la estabilidad mundial en el marco del TNP. La Conferencia de Examen de 2015 estará sometida probablemente a fuertes tensiones que pondrán a prueba la capacidad de adaptación del marco creado por el TNP al nuevo escenario internacional, en el que debería seguir siendo uno de sus pilares fundamentales.
\end{abstract}

Palabras clave: No Proliferación Nuclear, Tratado de no proliferación nuclear, energía nuclear, tecnología nuclear, zona libre de armas nucleares, Conferencia de Revisión del TNP.

Title in English: "The Treaty on Non-Proliferation of Nuclear Weapons: Key Issues in the Review Conference in 2015"

Abstract:

The nuclear non-proliferation regime in the last forty-five years was based in the Nonproliferation of Nuclear Weapons Treaty (NPT). Nowadays is has to face major challenges. In recent decades, several states have developed nuclear weapons, remaining outside the Treaty, while others have developed significant nuclear capabilities, leading to regional tensions. Moreover, a renaissance of nuclear energy has taking place in response to new energy needs, resulting in a growing development of nuclear technology in the world. Given the dual character, civil and military, of the nuclear technology, this process is an important challenge for the international community. It must preserve a balance between technical and economic progress and world stability under the NPT framework. The 2015 Review Conference will probably be exposed to strong tensions that will test the resilience and adaptation of the NPT regime to the new international scenario, in which it should remain one of its pillars.

Keywords: Nuclear Non-Proliferation, Non-Proliferation Treaty, nuclear energy, nuclear technology, nuclear weapons free zone, NPT Review Conference.

Copyright $@$ C UNISCI, 2015.

Las opiniones expresadas en estos artículos son propias de sus autores, y no reflejan necesariamente la opinión de UNISCI. The views expressed in these articles are those of the authors, and do not necessarily reflect the views of UNISCI.

\footnotetext{
${ }^{1}$ Gonzalo de Salazar Serantes es Doctor en Ciencias Políticas y Doctor en Ciencias de la Información. Ha sido previamente Subdirector General de No Proliferación y Desarme (Ministerio de Asuntos Exteriores y Cooperación), Miembro del Grupo Asesor sobre Seguridad Nuclear del Director General del Organismo Internacional de la Energía Atómica, Coordinador Internacional del Implementation \& Assessment Group de la Iniciativa Global contra el Terrorismo Nuclear, y Presidente de los Comités de No Proliferación y de Desarme de la Unión Europea durante la presidencia española en 2010.

E-Mail: gonzalo_desalazar@hotmail.com.
} 


\section{El Tratado de No Proliferación de Armas Nucleares}

El Tratado de No Proliferación de Armas Nucleares ${ }^{2}$ (TNP) constituye la piedra angular del régimen multilateral de no proliferación nuclear, y se basa en tres pilares que se refuerzan mutuamente: desarme, no proliferación y usos pacíficos de la energía nuclear. El tratado constituye actualmente el marco para mantener el equilibrio entre la seguridad internacional y el progreso técnico en este ámbito. En virtud de los artículos I, II y III del TNP los Estados dotados de armas nucleares (EEUU, Rusia, China, Reino Unido y Francia) se comprometen a no transferir armas nucleares a otros Estados, y los Estados sin armas nucleares se comprometen a no dotarse de ellas y a suscribir acuerdos de salvaguardias con el OIEA para que verifique que sus programas nucleares civiles no se desvían a fines militares.

Por otra parte, el artículo IV del TNP reconoce el derecho al uso de la energía nuclear con fines pacíficos y establece el principio de la cooperación internacional para el desarrollo de la energía nuclear.

En virtud del artículo VI del TNP, todos los Estados Partes se comprometen a llevar a cabo negociaciones relativas al cese de la carrera de armamentos nucleares y a un tratado de desarme general y completo bajo un estricto y efectivo control internacional.

Pueden destacarse tres problemas fundamentales en el régimen de no proliferación nuclear basado en el TNP:

- El tratado no es universal.

- Se ha producido una proliferación nuclear clandestina.

- El cumplimiento del compromiso de desarme nuclear aún no se ha materializado en su totalidad.

Hay países que, sin haber firmado el TNP, se han dotado de armas nucleares (India, Pakistán e Israel). Actualmente, mientras se producen avances significativos para establecer un acuerdo marco con Irán que garantice la finalidad pacífica de su programa nuclear, la preocupación de la comunidad internacional se centra en la República Democrática Popular de Corea.

\section{La Conferencia de Examen de 2015}

\subsection{Las Conferencias de Examen: antecedentes}

Con el fin de evaluar el cumplimiento de las disposiciones del TNP y fijar nuevos compromisos, se celebran cada 5 años Conferencias de Examen, cada una de ellas precedida de tres Comisiones preparatorias anuales. En las Conferencias de Examen confluyen temas vinculados a la seguridad internacional y a la amenaza nuclear:

- Universalidad.

- Condiciones para la retirada de un Estado Parte de conformidad con el artículo X del Tratado.

- Expediente nuclear de la República Democrática Popular de Corea.

- Expediente nuclear de Irán.

\footnotetext{
${ }^{2}$ Ver: Instrumento de 13 de diciembre de 1987, de Adhesión de España al Tratado sobre la no proliferación de las armas nucleares, hecho en Londres, Moscú y Washington el 1 de julio de 1968. BOE. Boletín Oficial del Estado núm. 313, 31 de Diciembre de 1987.
} 
- Resolución de 1995 a favor de la creación de una zona libre de armas nucleares y otras armas de destrucción masiva en Oriente Medio.

- Demanda de mayores esfuerzos a favor del desarme nuclear conforme al compromiso del Artículo VI.

- Entrada en vigor del Tratado de Prohibición Completa de Ensayos Nucleares (TPCEN).

- Negociación, aún pendiente, de un Tratado de prohibición de producción de material fisible para armas nucleares (TPMF).

Las cuatro últimas Conferencias de Examen del TNP han tenido una especial relevancia en este ámbito. Se pueden destacar los siguientes elementos:

1) En 1995 se alcanzó el acuerdo histórico de extender de forma indefinida la validez temporal del TNP. El compromiso se consiguió a cambio de la aprobación de una Resolución sobre Oriente Medio en la que se hace referencia a la existencia en la región de "instalaciones nucleares sin salvaguardias" y se insta al único Estado de la región que no es Parte del TNP (Israel) a firmar el Tratado. En Conferencias sucesivas, los países árabes han denunciado que no se ha hecho nada para la efectiva aplicación de esta Resolución.

2) En 2000 se aprobó un documento final consensuado que incluía "13 pasos prácticos" para avanzar en el proceso de desarme nuclear.

3) En 2005 la Conferencia concluyó sin un documento de consenso y habiendo consumido gran parte de su tiempo en discutir cuestiones de procedimiento ligadas a la agenda de la reunión, sin ningún avance sustantivo.

4) En 2010 se adoptó un Documento Final de la Conferencia por consenso, en el que se incluye un Plan de Acción ${ }^{3}$. Entre los objetivos políticos de este Plan de Acción habría que destacar la convocatoria de una conferencia en 2012 para negociar el establecimiento en Oriente Medio de una zona libre de armas nucleares y de otras armas de destrucción masiva, sentando así las bases de un proceso político que pueda servir para incrementar la estabilidad y la confianza mutua entre los países de la región.

Las cinco propuestas formuladas por el Secretario General de las NNUU en 2008 sirven aún como elementos del marco de referencia multilateral en materia de desarme y no proliferación nuclear:

- Aumento de las medidas efectivas, dirigidas especialmente al desarme de los países con armas nucleares, por medio de una convención con un instrumento de verificación o una red de acuerdos separados.

- Aumento de las garantías por parte de los miembros permanentes del CSNU respecto a los demás estados de no utilización o amenaza de utilización de armas nucleares.

- Entrada en vigor de TPCEN y negociación de un tratado de prohibición de producción de material fisionable.

- Un aumento de la información puesta a disposición por parte de los estados con armas nucleares.

\footnotetext{
${ }^{3}$ NPT RevCon Final Document- NPT/CONF.2010/50.
} 
- Medidas complementarias, como la eliminación de otros tipos de armas de destrucción masiva, o medidas generales contra el terrorismo.

No obstante, en los últimos años se ha producido una desaceleración del proceso de desarme frente a una agenda internacional más centrada en la no proliferación, por lo que entre los países neutrales y no alineados se extiende la convicción de que no existe una verdadera intención de avanzar hacia un mundo sin armas nucleares. También hay tensiones derivadas de la proliferación nuclear en Oriente Medio y en Asia oriental, y no se ha producido la convocatoria de una Conferencia para la creación de una Zona Libre de Armas Nucleares y de otras Armas de Destrucción Masiva en Oriente Medio, mientras continua la parálisis de la Conferencia de Desarme.

Estos factores han motivado un cierto pesimismo en gran parte de la comunidad internacional de cara a los resultados de la Conferencia de Examen de 2015, mientras se desarrollan nuevas iniciativas cuyo desenlace a largo plazo es difícil de prever: las conferencias sobre el impacto humanitario de las armas nucleares y la creación de un grupo de trabajo sobre el desarme nuclear fuera de la Conferencia de Desarme, sin participación de las potencias nucleares.

\subsection{Las perspectivas para la Conferencia de Examen de 2015: los temas clave}

En la actualidad, los intentos de limitar la proliferación nuclear se centran en el mantenimiento de un equilibrio adecuado entre los tres pilares del Tratado de No Proliferación de Armas Nucleares: el desarme nuclear, los mecanismos de no proliferación y la cooperación para usos pacíficos de la energía nuclear. El TNP ha permitido a lo largo de su existencia retardar en gran medida la proliferación. Si en 1968 se estimaba que a principios de este siglo habría más de 20 potencias nucleares, lo cierto es que actualmente sólo hay 9 Estados con capacidad nuclear militar.

Como se ha indicado anteriormente, el interés en la energía nuclear con fines civiles en la era post-Fukushima - en el que se ha revalorizado el concepto de seguridad tecnológica - , el proceso de difusión de materiales y de tecnologías sensibles asociadas al sector nuclear, y las aplicaciones de doble uso de muchas de ellas, constituyen rasgos del escenario internacional que implican nuevos retos.

Tecnologías sensibles que tienen su origen en países industrializados occidentales se han difundido mediante el comercio legítimo, el tráfico ilícito o la fabricación ilícita de réplicas en otras zonas del planeta. También ha habido un fenómeno de desarrollo autóctono de ciertas tecnologías en algunos países, muchas veces basadas en conocimientos técnicos y equipos obtenidos de los suministradores más avanzados. Además de incorporarse al mercado nuevos productores de esas tecnologías, se ha desarrollado una nueva red comercial por la que fluyen estos materiales, fuera del alcance de los mecanismos multilaterales de control de exportaciones, especialmente del Grupo de Suministradores Nucleares.

Pero en el momento actual no sólamente hay nuevos desafíos, sino que hay también oportunidades para superarlos: el riesgo de una confrontación nuclear global ha disminuido considerablemente y hoy es muy improbable. Las dos grandes potencias nucleares, Rusia y Estados Unidos, no sólo han emprendido un proceso de reducción de sus arsenales desde 1991, sino que han firmado un nuevo tratado de desarme nuclear - actualmente en aplicación - que reduce en un 30\% más sus arsenales, el Nuevo START. 
En la balanza positiva, también puede citarse el acuerdo alcanzado por el grupo UE $3+3$ e Irán sobre los parámetros de una negociación sobre su programa nuclear, que abre nuevos interrogantes y perspectivas para los próximos meses.

En cuanto a las nuevas iniciativas multilaterales (conferencias sobre el impacto humanitario de las armas nucleares y creación de un grupo de trabajo sobre el desarme nuclear fuera de la Conferencia de Desarme), puede decirse que los estados promotores parten de la premisa de la parálisis de la maquinaria de desarme de Naciones Unidas. Se pretende con ellas adoptar en el ámbito nuclear el modelo de las convenciones de Ottawa -minas antipersonales- y Oslo -municiones en racimo. Desde el punto de vista político, ambos instrumentos han logrado el propósito de crear un "paradigma moral" en el ámbito del desarme humanitario, y a la vez un estigma en torno al uso de esas armas. Dicho proceso conlleva también cuestionar ciertos aspectos relacionados con las armas nucleares: su papel en las doctrinas militares, inversiones en modernización de arsenales y costes de mantenimiento, entre otros. El proceso no cuenta con la participación de los estados dotados de armas nucleares, aunque algunos de ellos han estado presentes en la conferencia de Viena de 2014 sobre el impacto humanitario. En todo caso, cabe la posibilidad de que afecte de alguna manera en el futuro al régimen actual de no proliferación nuclear.

En la Conferencia de Examen, los debates sobre todos estos temas se estructurarán, como es habitual, en los tres pilares del TNP: desarme, no proliferación y usos pacíficos.

\subsection{Desarme}

El contenido de la Resolución 1887 del Consejo de Seguridad de Naciones Unidas y el nuevo acuerdo START entre Estados Unidos y Rusia reflejaron en 2010 un nuevo impulso al desarme. En su famoso "discurso de Praga" de abril de 2009, el presidente de EEUU lanzó varias iniciativas de desarme nuclear y prevención de la proliferación que han marcado el ritmo de la agenda internacional en los últimos años. Los hitos más importantes de esta política en 2010 han sido el acuerdo de desarme nuclear firmado y ratificado por EEUU y Rusia, llamado "Nuevo START" 4 , las Cumbres de Seguridad Física Nuclear, el lanzamiento de un programa de trabajo internacional de la Iniciativa Global para Combatir el Terrorismo Nuclear (IGTN), y la inclusión de la no proliferación entre las prioridades de la OTAN en el Nuevo Concepto Estratégico de la Alianza.

\subsubsection{Reducción de armamentos}

La propuesta planteada en 2013 por EE.UU a Rusia de reducción de un tercio de las armas nucleares estratégicas desplegadas (desde el límite actual de 1.550 fijado por el Nuevo START hasta un nuevo límite de 1.000-1.100 cabezas nucleares) no sólo supone tomar la iniciativa hacia un nuevo paso en el proceso de reducción de los arsenales nucleares de ambas potencias, sino que también tiene implicaciones en las políticas de seguridad y defensa de otros países 5 .

\footnotetext{
${ }^{4}$ Según los datos hechos públicos por el Departamento de Estado de EEUU, Rusia tenía 1.643 cabezas nucleares desplegadas en 528 vectores el 1 de enero de 2015. En esa fecha EEUU tenía 1642 cabezas nucleares, desplegadas en 794 vectores. Las cifras totales de vectores estratégicos desplegados y no desplegados eran en esa fecha 911 para Rusia y 912 para EEUU. Véase US Department of State: "New START Treaty Aggregate Numbers of Strategic Offensive Arms", en http://www.state.gov/t/avc/newstart/c39906.htm.

5 The White House: "Nuclear Weapons Employment Strategy of the United States", Fact Sheet, Office of the Press secretary, Washington (19 de junio de 2013). Ver también The White House: "Remarks by President Obama at the Brandenburg Gate", Office of the Press secretary, Berlin (19 de junio de 2013), p. 5.
} 
Mientras tanto, en Rusia se reflexiona no sólo sobre posibles negociaciones de desarme nuclear "bilaterales cruzadas y complementarias" como un formato posible con modelos definidos en torno al concepto de "relación de disuasión nuclear" (frente al nada realista formato multilateral simultáneo a 5 o a 9), sino también sobre la base de una visión integrada de la seguridad que incluiría el armamento convencional y otros activos estratégicos. ${ }^{6}$ Desde esta perspectiva, las armas nucleares se consideran en Rusia como un elemento más del equilibrio militar global, y no se pueden analizar o reducir al margen de otros elementos:

- Defensa antimisiles

- Armas estratégicas convencionales

- Armas desplegadas en el espacio

- Fuerzas convencionales

Así, frente a la nueva propuesta de EEUU de reducir en un 30\% las armas nucleares estratégicas desplegadas y de abordar una negociación de reducción de armas nucleares tácticas, Rusia considera que una negociación sobre desarme nuclear debe incluir también otros factores, asociando el desarme nuclear con el escudo antimisiles, el espacio ultraterrestre y el control de armamentos convencionales. Sin embargo, el clima político que impera actualmente en las relaciones internacionales no favorece el impulso de nuevas negociaciones a corto plazo.

Todos estos planteamientos están aún lejos de materializarse en propuestas concretas, y el P-5 ha optado por preservar el status quo.

A pesar de los avances realizados en años anteriores por los Estados dotados de armas nucleares en las reducciones de armamento nuclear, los países no alineados y neutrales exigen esfuerzos mayores, incluyendo una modificación de las doctrinas militares basadas en el posible recurso a las armas nucleares. En particular, los países de la llamada Coalición de la Nueva Agenda (Nueva Zelanda, Brasil, México, Egipto, Irlanda, Suecia y Sudáfrica) son muy críticos con lo que consideran una falta de progreso en el desarme nuclear, reducido prácticamente al cumplimiento del nuevo Tratado START entre EEUU y Rusia.

Por su parte, Ucrania ha cuestionado la validez de los acuerdos de desarme posteriores a la guerra fría, al considerar que Rusia, con su intervención en Ucrania en 2014, ha incumplido el Memorando de Budapest por el que otorgaba seguridades a su integridad territorial a cambio de ceder sus armas nucleares.

\subsubsection{Seguridades negativas}

Algunos países sin armas nucleares han venido exigiendo garantías de no utilización de armas nucleares contra sus territorios por parte de las potencias nucleares (seguridades negativas).

\footnotetext{
${ }^{6}$ La última versión de la Doctrina Militar rusa, adoptada el 26 de diciembre de 2014, mantiene un enfoque similar a la de 2010. También señala a la OTAN como una amenaza, y fija como prioridades el desarrollo de una defensa antimisiles, de un dispositivo ofensivo con alcance global, de sistemas convencionales de alta precisión y la defensa de sus intereses en el Ártico. La Doctrina militar de 2014 considera que la OTAN ha emprendido un proceso de rearme y se extiende hacia las fronteras rusas. También aborda la amenaza derivada del uso de tecnologías de la comunicación y de la información como instrumentos de desestabilización interna, lo que requiere, según sus autores, una respuesta. Ver

http://www.globalsecurity.org/military/world/russia/doctrine.htm;

http://carnegie.ru/eurasiaoutlook/ ?fa=57607.
} 
La Resolución 984 (1995) del Consejo de Seguridad recoge las garantías otorgadas por las cinco potencias nucleares, aunque no son jurídicamente vinculantes ni incluyen la amenaza del uso de armas nucleares, como reclaman los países No Alineados.

También se ha dado continuidad en los últimos años a los esfuerzos para la creación de zonas libres de armas nucleares establecidas sobre la base de acuerdos libremente aceptados por los estados de cada región con la creación de la ZLAN de Asia Central basada en el Tratado de Semipalatinsk ${ }^{7}$. A través de las Zonas Libres de Armas Nucleares se incorporaron anejos a dichos tratados que debían suscribir las potencias nucleares y que incluían seguridades negativas.

Los países no alineados y los de la Coalición de la Nueva Agenda solicitan compromisos jurídicamente vinculantes en relación con las seguridades negativas al empleo de armas nucleares contra los Estados no dotados de dichas armas. La negociación de un instrumento internacional jurídicamente vinculante que otorgue garantías a los Estados Partes del TNP contra la amenaza o el uso de armas nucleares es una demanda que reaparece en todas las conferencias y reuniones sobre desarme nuclear.

\subsubsection{Gradualismo y abolicionismo}

Desde 1970 ha prevalecido una corriente gradualista del desarme nuclear en el marco del TNP, que prevé un proceso gradual reflejado en las trece medidas incluidas en el informe de la Conferencia de Examen de 2000. Frente a lo que muchos países no alineados y neutrales califican de "estancamiento", desde 2013 ha surgido con fuerza una corriente internacional a favor de la ilegalización de las armas nucleares, con apoyo de los movimientos pacifistas, del mismo modo que se ha hecho con las armas químicas y biológicas. Un "Comunicado Conjunto" sobre esta opción presentado por Nueva Zelanda en la Primera Comisión de la $69^{\text {a }}$ sesión de la AGNU fue suscrito por 155 Estados. Las dos iniciativas complementarias ya citadas han abierto en 2013 una nueva fase en este proceso:

- Se han celebrado tres conferencias sobre el Impacto Humanitario de las Armas Nucleares ${ }^{8}$ : Oslo, 2013; Nayarit (México), 2014; y Viena, 2014.

- Por otra parte, la Resolución 67/56 AGNU ${ }^{9}$, presentada también por Austria, México y Noruega, establecía en Ginebra un grupo de trabajo abierto sobre desarme nuclear, que no contó con el apoyo de las potencias nucleares, en el que se enfrentaban la posición gradualista de los países occidentales al "abolicionismo" de los países neutrales y no alineados.

\footnotetext{
7 "Zona Libre de Armas Nucleares de Asia Central", basada en el Tratado de Semipalatinsk de 2006, con entrada en vigor en 2009. Tiene 5 Estados Parte. Los cinco estados nucleares Partes del TNP han suscrito el Protocolo del Tratado. Ver http:// disarmament.un.org/ treaties/t canwfz/text.

${ }^{8}$ Dicho enfoque desarrolla en cierto modo el controvertido punto de la "Opinión consultiva de la Corte Internacional de Justicia sobre la legalidad de la amenaza o el empleo de armas nucleares" en relación con su impacto humanitario, de 1996, en la que, gracias al voto de calidad del Presidente, el tribunal declaró que la Corte no podía pronunciarse definitivamente sobre si la amenaza o el empleo de las armas nucleares sería lícito o ilícito en circunstancias extremas de legítima defensa, en las que corriera peligro la propia supervivencia de un Estado: "Opinión consultiva de la Corte Internacional de Justicia sobre la legalidad de la amenaza o el empleo de armas nucleares" $\mathrm{A} / 51 / 218$, (19 de julio de 1996), tema 71 del programa provisional, en http://www.icjcij.org/homepage/sp/advisory/advisory_1996-07-08.pdf.

${ }^{9}$ A/RES/67/56. "Promoción de las negociaciones multilaterales de desarme nuclear", Acta A/67/PV.48 3 diciembre 2012, en http://www.un.org/es/ga/67/resolutions.shtml.
} 


\subsection{No Proliferación}

Los fundamentos de un régimen global de prevención y los casos de proliferación regional han sido el centro de atención en el debate sobre este pilar. Muchos estados destacan principalmente el incumplimiento de las obligaciones derivadas del TNP por parte de la República Democrática Popular de Corea (cuya retirada del Tratado no se reconoce), de Irán y de Siria. Temas importantes del pilar "no proliferación" del TNP son la definición del estándar internacional de verificación, Oriente Medio, las medidas para evitar la proliferación nuclear entre actores no estatales y las condiciones de retirada del Tratado.

\subsubsection{Verificación}

De cara al futuro es importante definir un estándar internacional de verificación, que para una mayoría debe estar basado en el modelo de Acuerdo de Salvaguardias Globales y el Protocolo Adicional.

En este contexto, la mayoría de los Estados Parte destaca el importante papel que desempeña el OIEA y su sistema de salvaguardias, aunque hay divergencias sobre el papel y el estatus del Protocolo Adicional a los Acuerdos de Salvaguardias, que una mayoría considera elemento fundamental del estándar de verificación. Todos los Estados son partidarios de la universalización del Tratado, pero los países no alineados se concentran principalmente en el caso de Israel, pasando por alto los casos de India y Pakistán.

\subsubsection{Actores no estatales}

Otro desafío importante debatido es la forma de evitar los riesgos de desviación de tecnologías sensibles hacia actores no estatales con fines terroristas. En este sentido, la Resolución 1540 adoptada en 2004 por el Consejo de Seguridad es el marco en el que deben desarrollarse medidas para prevenir que terroristas puedan adquirir materiales nucleares o radioactivos con los que puedan llevar a cabo atentados de destrucción masiva. Esto implica fortalecer la seguridad nuclear, actividad en la que el papel del Organismo Internacional de la Energía Atómica es fundamental.

\subsubsection{Oriente Medio}

No ha sido posible convocar en 2012 la Conferencia sobre la Zona Libre de Armas Nucleares y otras Armas de Destrucción Masiva en Oriente Medio ${ }^{10}$, prevista desde 1995 e incluida en el Plan de Acción de 2010. Este es un tema controvertido desde los años 70, que nuevamente marcará las tensiones en la Conferencia de Examen de $2015^{11}$. Egipto y otros países árabes denuncian que no se han producido avances para la puesta en práctica de la Resolución de 1995 y, especialmente, que no se realiza suficiente presión sobre Israel para que se adhiera al TNP y someta todas sus instalaciones nucleares a las salvaguardias del OIEA. Por otra parte, Egipto y Siria no han firmado la Convención de Prohibición de Armas Químicas, mientras que Israel sí la ha firmado, pero no la ha ratificado todavía.

\footnotetext{
${ }^{10}$ En la Conferencia de Extensión del TNP de 1995 se aprobó una Resolución sobre Oriente Medio apoyando la creación de una zona libre de armas de destrucción masiva en Oriente Medio. Según Egipto y otros países árabes, la aprobación de esta Resolución sobre Oriente Medio en el marco del TNP fue una condición imprescindible para que diesen su consentimiento a la extensión indefinida del TNP a partir de 1995 (el TNP tenía una validez inicial de 25 años y tras ese plazo debía adoptarse una nueva decisión sobre su extensión).

${ }^{11}$ Desde 1974 se aprueba cada año una Resolución de la Asamblea General de NN.UU. sobre una zona libre de armas nucleares y de otras armas de destrucción masiva en Oriente Medio (en última instancia, Resolución AGNU 63/38), incluso con el apoyo de Israel que, sin embargo, reitera cada vez que no será posible alcanzar dicho objetivo hasta que haya avances hacia una paz justa, duradera y global en la región. Esta zona incluiría también otras armas de destrucción masiva como las químicas y biológicas, así como sus vectores de lanzamiento.
} 
Una cuestión central en Oriente Medio es el programa nuclear iraní y las perspectivas de solución abiertas por el acuerdo alcanzado el pasado 2 de abril sobre los parámetros de la negociación por el grupo UE $3+3$.

El 24 de noviembre de 2013, Irán y el UE 3+3 adoptaron el "Joint Plan of Action" 12 (JPA) sobre el programa nuclear iraní, que tenía como objetivo lograr un acuerdo político para alcanzar una solución global que garantizase que el programa nuclear iraní tiene carácter exclusivamente pacífico, y permitiera el levantamiento de las sanciones impuestas a Irán por el Consejo de seguridad de Naciones Unidas, por EEUU y por la $\mathrm{UE}^{13}$. De forma paralela, el OIEA e Irán establecieron un "Marco para la Cooperación" de carácter técnico, para resolver las cuestiones pendientes del programa nuclear. La dificultad en aclarar las Posibles Dimensiones Militares del programa nuclear, dada la escasa cooperación de las autoridades iraníes, ha sido el principal obstáculo hasta ahora en este proceso.

El acuerdo alcanzado por el UE $3+3$ con Irán el 2 de abril ${ }^{14}$ se basa en una serie de parámetros que deben ser negociados hasta el 30 de junio, para limitar el tiempo requerido desde el punto de vista técnico para conseguir el material fisible necesario para la fabricación de un arma nuclear a un mínimo de 12 meses, durante los próximos 10 años:

- Irán se compromete a no enriquecer uranio por encima del $3.67 \%$.

- No se construirán nuevas instalaciones de enriquecimiento en 15 años.

- Reducirá el número de centrifugadoras instaladas hasta 6.104 unidades (a partir de unas 19.000 que tiene actualmente, solamente 5.060 seguirán enriqueciendo uranio durante los próximos 10 años, y el resto hasta 6.104 se mantendrán para investigación y desarrollo).

- Irán se compromete a no utilizar la instalación de Fordow para enriquecer uranio durante 15 años.

- Las capacidades de la instalación de Natanz se limitarán a 5.060 centrifugadoras del tipo IR-1 durante los próximos 10 años. Los modelos de centrifugadoras IR-2, IR-4, IR6 o IR-8, más modernos, no se utilizarán durante 10 años.

- Irán conservará sólo 300 kgs de uranio enriquecido al 3.67\% (frente a los 10.000 kgs actuales).

- Irán se compromete a cumplir el Protocolo Adicional al Acuerdo de salvaguardias, dando al OIEA acceso a todas las instalaciones nucleares.

- Irán se compromete a aplicar el Código Modificado 3.1 del Acuerdo de salvaguardias, que requiere la notificación previa de la construcción de cualquier nueva instalación.

- Irán debe aplicar las medidas establecidas por el OIEA para esclarecer las cuestiones pendientes sobre las Posibles Dimensiones Militares.

- Irán procederá a rediseñar el reactor de Arak, para que no pueda producir plutonio.

\footnotetext{
12 "Joint Plan of Action", Geneva, 24 de noviembre de 2013, en http://www.eeas.europa.eu/statements/docs/ 2013/ 13112403 en.pdf.

${ }^{13}$ Proceso iniciado el 20 de enero de 2014, prorrogado el 24 de noviembre de 2014 hasta el 30 de junio de 2015 para lograr un acuerdo final, precedido por un acuerdo político a finales de marzo que finalmente ha sido alcanzado el 2 de abril. Durante este tiempo se ha mantenido bajo la vigilancia del OIEA el programa nuclear iraní en los términos previstos en el JPA.

14 "Parameters for a Joint Comprehensive Plan of Action regarding the Islamic Republic of Iran 's Nuclear Program", Lausanne, 2 de abril de 2015.
} 
- Las sanciones serán suspendidas cuando se verifique el cumplimiento de las medidas descritas. Para ello, se aprobará una nueva Resolución del CSNU que aplique las disposiciones del nuevo acuerdo. En caso de incumplimiento por Irán, las sanciones volverán a estar en vigor.

Este acuerdo es un paso significativo hacia un acuerdo global sobre la cuestión nuclear iraní, con muchos aspectos positivos, ya que ha permitido un diálogo constructivo con Estados Unidos y limita el programa nuclear iraní, sobre el que establece mayor control y transparencia bajo supervisión del OIEA, incluyendo el esclarecimiento de las posibles dimensiones militares como una condición para el levantamiento de sanciones. Sin embargo, quedan aún muchas cuestiones pendientes no solo para alcanzar un acuerdo definitivo el 30 de junio, sino también para su aplicación en una fase ulterior. Todas ellas son de gran importancia para los países de la región, muchos de los cuales han mostrado su inquietud por el resultado parcial de las negociaciones. En este, sentido, será preciso abordar la complejidad del sistema de sanciones para su suspensión progresiva, que requiere, entre otras cosas, una nueva resolución del CSNU.

\subsubsection{Artículo X}

Finalmente, las condiciones de retirada de un Estado del Tratado constituyen otro tema polémico, ya que no hay acuerdo sobre la interpretación y el alcance de dicho artículo. Para muchos estados no alineados no es necesario discutir esta cuestión, ya que solamente hay un caso de retirada (Corea del Norte), y poner condiciones a la retirada del Tratado en virtud del artículo X sería inaceptable a menos que hubiese una enmienda del Tratado, que no se ha producido. Para otros estados, especialmente los occidentales, la retirada debería tener un efecto retroactivo de desmantelamiento de las instalaciones nucleares construidas al amparo del artículo III del Tratado.

\subsection{Usos pacíficos de la energía nuclear}

El derecho a la utilización de la energía nuclear para fines pacíficos (de conformidad con el Artículo IV del TNP), es un tema clave en un momento de renovado interés por parte de numerosos países para desarrollar su capacidad de producir energía a gran escala, dados los riesgos de proliferación que puede conllevar, incluyendo la amenaza de que material nuclear llegue a manos de grupos terroristas.

Los países en desarrollo solicitan mayor apoyo para la cooperación técnica destinada a los usos pacíficos, incluida la que se presta a través del OIEA. También reclaman que se eliminen los obstáculos establecidos a las transferencias de tecnología a través de los regímenes de control de exportaciones, que consideran un obstáculo para el desarrollo de los usos pacíficos.

Por otra parte, la creación de un banco de uranio de bajo enriquecimiento para el combustible nuclear en el marco del OIEA, apoyado por los países occidentales como un paso decisivo para consolidar los enfoques multilaterales del ciclo del combustible nuclear, también es un tema en el que se manifiestan divergencias. Los esfuerzos para crear mecanismos multilaterales que aseguren el suministro de combustible nuclear a cualquier país que lo necesite, sin necesidad de establecer mecanismos nacionales de enriquecimiento de uranio y evitando los riesgos de desvío a programas militares que ello conllevaría, es un tema controvertido. Muchos Estados apoyan el concepto de desarrollo responsable de los usos pacíficos de la energía nuclear basándose en los conceptos de seguridad, no proliferación y enfoques multilaterales del ciclo del combustible nuclear. Cada país tiene el derecho a definir su propia estrategia energética, basada en el derecho inalienable a los usos pacíficos 
reconocido por el TNP en el artículo IV. Pero esto requiere los más elevados estándares de seguridad, de no proliferación y de protección física, en los que pueden contar con la asistencia del OIEA.

\section{La posición de la UE}

La posición común de la UE sobre el TNP ha sido generalmente un mínimo común denominador que intenta conciliar los intereses de grupos de países con intereses diferentes:

- Francia y Reino Unido (potencias nucleares);

- Suecia e Irlanda, países neutrales y fuertemente comprometidos con el desarme nuclear $^{15}$;

- Austria, que se opone a cualquier postura que favorezca la promoción de la energía nuclear;

- El grupo mayoritario formado por países con posturas intermedias, que tratan de buscar un equilibrio entre los usos pacíficos de la energía nuclear y la estabilidad global.

La Unión Europea, que desempeña un papel muy destacado como contribuyente principal a los programas de cooperación del OIEA, ha dado habitualmente prioridad a ciertos aspectos del ciclo de examen del TNP. En primer lugar, reafirma el compromiso de los Estados Parte con sus obligaciones y apoya la universalidad del Tratado. También ha buscado fortalecer su aplicación mediante medidas concretas consensuadas en los tres pilares, incluida la creación de una zona libre de armas nucleares y de otras armas de destrucción masiva en Oriente Medio.

La UE destaca su compromiso con los procesos de desarme. Entre ellos se incluye el apoyo a la entrada en vigor del Tratado de Prohibición Completa de Ensayos Nucleares y su posición a favor del inicio de negociaciones sobre un Tratado de Prohibición de Producción de Material Fisible en la Conferencia de Desarme.

Respecto del TNP en general, la UE desea que se avance de forma equilibrada en los tres pilares: no proliferación, desarme y cooperación en usos pacíficos de la energía nuclear. Los objetivos de la UE en este proceso se plasman en la Decisión del Consejo sobre la posición común para la Conferencia de Examen del TNP en $2010^{16}$ :

- La Unión Europea desea cooperar con la comunidad internacional para reafirmar los principios fundamentales del Tratado como piedra angular del régimen de no proliferación.

- Esta política se basa en un enfoque equilibrado entre los tres pilares del Tratado, desarme, no proliferación y usos pacíficos, mediante medidas pragmáticas y consensuadas para impulsar los esfuerzos internacionales en esta dirección.

- La Unión Europea reafirma su compromiso de lograr un mundo más seguro y crear las condiciones para un mundo sin armas nucleares.

\footnotetext{
${ }^{15}$ Suecia e Irlanda son miembros de la New Agenda Coalition junto con países como Egipto, Sudáfrica, Brasil, México y Nueva Zelanda.

${ }^{16}$ Decisión 2010/212/PESC del Consejo, de 29 de marzo de 2010, relativa a la posición de la Unión Europea en la Conferencia de las Partes del año 2010 encargada del examen del Tratado sobre la no proliferación de las armas nucleares. Diario Oficial de la Unión Europea de 10 de abril de 2010. Esta Decisión del Consejo fue elaborada y adoptada durante la presidencia española de la UE en 2010.
} 
Las Conclusiones del Consejo de Asuntos Exteriores de la UE del 20 de abril ${ }^{17}$ actualizan los elementos de esta posición común, entre los que se incluye una referencia a las graves consecuencias del uso de las armas nucleares, reflejando el debate abierto sobre el impacto humanitario.

La UE considera que el Tratado sobre la No Proliferación de Armas Nucleares es la piedra angular del sistema mundial de no proliferación nuclear, la base fundamental para la consecución del desarme nuclear de conformidad con el artículo VI del TNP y una parte importante para el futuro desarrollo de las aplicaciones de la energía nuclear para fines pacíficos. Su objetivo es reforzar el sistema internacional de no proliferación nuclear fomentando un resultado sustancial y equilibrado de la Conferencia de Examen del Tratado para lograr un avance tangible y realista hacia los objetivos consagrados en el TNP. Sin embargo, en la UE se ponen de manifiesto también las diferentes opiniones en torno al debate entre gradualismo y abolicionismo, este último defendido por Austria e Irlanda.

\section{Perspectivas del régimen de no proliferación nuclear}

Actualmente nos encontramos en una "pausa estratégica" en la que existe un equilibrio, pero éste empieza a ser frágil. Los sistemas de armas nucleares son complejos desde el punto de vista organizativo, técnico e industrial, y evolucionan en ciclos largos impulsados por decisiones prácticas y también por inercia. La evolución real del contexto geopolítico es más rápida que ese proceso de adopción de decisiones, conduciendo a desfases entre los sistemas de disuasión y las amenazas reales existentes. Sin embargo, la estabilidad estratégica no se basa sólo en los números, sino también en la distensión, la transparencia, y el respeto recíproco basado en los principios recogidos por la Carta de las Naciones Unidas, fundamento de la confianza mutua.

China podría ser en el futuro un factor decisivo en la evolución de ese equilibrio. A pesar de su importante desarrollo militar y tecnológico en las últimas décadas, persiste la disparidad militar y estratégica con EE.UU. Actualmente es preciso subrayar el escaso conocimiento que se tiene de su arsenal nuclear, que se estima en unas 250-300 cabezas nucleares. Desde el punto de vista de Rusia y de China, será necesario preservar un equilibrio estratégico global con EEUU, lo que influirá en futuros compromisos de desarme nuclear.

Por otra parte, muchas cuestiones importantes para la no proliferación nuclear han quedado fuera del Plan de Acción de 2010, aunque están incluidas en el informe del presidente de la Conferencia de 2010, en el que se refleja el estado de los debates. En este ámbito se pueden destacar las divergencias que se han puesto de manifiesto entre los Estados Partes del Tratado sobre diversas cuestiones que seguirán en la agenda en 2015:

- El papel del Protocolo Adicional de los Acuerdos de Salvaguardias como parte integrante de un estándar de verificación reforzado.

\footnotetext{
17 "The Council notes the severe consequences associated with nuclear weapons use and emphasizes that all States share the responsibility to prevent such an occurrence from happening. The Council further notes, in this respect, the ongoing discussions on the consequences of nuclear weapons, in the course of which different views are being expressed, including at an international conference organized by Austria, in which not all EU Member States participated" (Council conclusions on the Ninth Review Conference of the Parties to the Treaty on the Non-Proliferation of Nuclear Weapons). "Council of the European Union Luxemburg", Outcome of the Council Meeting, 3382th meeting, 8084/15. Foreign Affairs, Press, Brussels: General Secretariat of the Council (20 de abril de 2015), p.10.
} 
- El alcance de los mecanismos de control de exportaciones, efectivo y transparente, para que no restrinjan el comercio de tecnología para fines pacíficos de la energía nuclear.

- La conveniencia de elaborar un instrumento jurídicamente vinculante que prohíba los ataques o amenazas de ataque sobre instalaciones nucleares para fines pacíficos.

- La demanda de un elevado número de países de que se establezca un marco legal y un calendario preciso para el desarme nuclear de conformidad con el artículo VI del Tratado.

- Las distintas interpretaciones sobre la Opinión Consultiva de la Corte Internacional de Justicia sobre la legalidad de la amenaza o el uso de las armas nucleares (1996).

Los defensores de la prohibición de las armas nucleares con un nuevo tratado mantienen que ningún Estado ni organización internacional tiene medios para hacer frente a las emergencias humanitarias y la consecuencias a largo plazo causadas por una detonación nuclear ocurrida en un área poblada, ni para facilitar la asistencia adecuada a los afectados. También consideran que el sufrimiento causado a la población por la utilización de las armas nucleares no es sólo una cuestión legal, sino que necesita un enfoque ético que trascienda las interpretaciones sobre su legalidad.

Por otra parte, hay distintos análisis sobre el derecho a retirarse del Tratado, establecido en el artículo X. Muchos Estados - especialmente los suministradores de tecnología - señalan que el Estado Parte que se retira del TNP, además de ser responsable por las violaciones cometidas antes de la retirada, debería mantener bajo salvaguardias las instalaciones nucleares obtenidas mediante importación de tecnología cuando era Parte del Tratado. También señalan la necesidad de incluir cláusulas de desmantelamiento y devolución de dichas tecnologías en caso de retirada. La mayoría de los países no alineados rechaza este enfoque.

En definitiva, en el escenario actual en vísperas de la Conferencia de Examen del TNP existen divergencias sobre la interpretación del TNP y la forma en la que ha sido cumplido en las últimas décadas, mientras surgen nuevos desafíos relacionados con el desarrollo del sector energético civil, el comercio mundial y la emergencia de nuevas potencias económicas y tecnológicas. La proliferación nuclear horizontal ha evolucionado en el marco de los avances tecnológicos y las transacciones realizadas en las últimas décadas, pero sobre todo como consecuencia de motivaciones estratégicas de determinados países en aras de su seguridad nacional o de ambiciones hegemónicas, sometiendo a fuertes tensiones al régimen de no proliferación basado en el TNP desde 1970.

Actualmente es difícil saber si esta tendencia puede a cambiar. Conflictos latentes en distintas regiones del mundo - estratégicos o ideológicos - han contaminado gradualmente las tensiones vinculadas a la proliferación nuclear. El proceso podría alcanzar incluso a las formas de enfrentamiento asimétrico con actores no estatales.

Este escenario, en el que se superpone la creciente demanda energética de las economías emergentes y la necesidad de mitigar el cambio climático reduciendo las emisiones de carbono, incluye actualmente importantes contradicciones que será necesario resolver con la cooperación de toda la comunidad internacional, ya que también están en juego, no sólo legítimos intereses económicos, sino también la paz.

Se trata de un proceso extremadamente delicado en que es preciso mantener un equilibrio entre los tres pilares del TNP antes citados y desarrollar nuevos mecanismos que 
permitan aumentar la confianza mutua, dando nuevos pasos hacia el desarme y haciendo que el proceso gradual que hemos defendido desde 1970 no se detenga.

Hay una serie de cuestiones que habría que abordar en un futuro próximo para impulsar este proceso. En primer lugar, es preciso un esfuerzo colectivo para cumplir el Plan de Acción incluido en el documento final de la Conferencia de Examen del TNP de 2010, manteniendo el diálogo abierto en la negociación multilateral sobre todos aquellos puntos discutidos que quedan abiertos. Entre ellos destaca el movimiento sobre el impacto humanitario de las armas nucleares, que merece una reflexión no sólo por su alcance político, sino también por la importancia ética del problema que plantea.

También es fundamental impulsar nuevas iniciativas: los preparativos para celebrar una conferencia sobre la creación de una zona libre de armas nucleares y de otras armas de destrucción masiva en Oriente Medio, contando con todos los países de la región; iniciar la negociación sobre un Tratado de Prohibición de Producción de Material Fisible en la Conferencia de Desarme; impulsar la entrada en vigor del Tratado de Prohibición Completa de Ensayos Nucleares, o al menos su aplicación provisional. Será preciso tender puentes entre los distintos enfoques sobre todas estas cuestiones.

Finalmente, es necesario reforzar el papel del Organismo Internacional de la Energía Atómica como garante de ese delicado equilibrio entre la cooperación en el ámbito de los usos pacíficos de la energía nuclear y la no proliferación, lo que requeriría igualmente avanzar hacia la consolidación de un estándar de verificación universalmente aceptado, basado en los Acuerdos de Salvaguardias Globales y el Protocolo Adicional, como corolario de la transparencia multilateral y de la confianza mutua. 